\title{
Regional brain changes in patients with diabetic optic neuropathy: a resting-state functional magnetic resonance imaging study
}

\author{
Si-Yi Chen ${ }^{1 \#}$, Guo-Qian Cai ${ }^{2 *}$, Rong-Bin Liang ${ }^{1 \#}$, Qi-Cheng Yang ${ }^{3}$, You-Lan Min ${ }^{1}$, Qian-Min Ge ${ }^{1}$, \\ Biao Li ${ }^{1}$, Wen-Qing Shi ${ }^{1}$, Qiu-Yu Li ${ }^{1}$, Xian-Jun Zeng ${ }^{2}$, Yi Shao ${ }^{1}$ \\ ${ }^{1}$ Department of Ophthalmology, the First Affiliated Hospital of Nanchang University, Nanchang, China; ${ }^{2}$ Department of Radiology, the First \\ Affiliated Hospital of Nanchang University, Nanchang, China; ${ }^{3}$ The Chinese University of Hong Kong, Shatin, New Territories, Hong Kong, China \\ \#These authors have contributed equally to this work.
}

Correspondence to: Yi Shao. Department of Ophthalmology, The First Affiliated Hospital of Nanchang University, No. 17, Yongwaizheng Street, Donghu District, Nanchang 330006, China. Email: freebee99@163.com; Xian-Jun Zeng. Department of Radiology, The First Affiliated Hospital of Nanchang University, No. 17, Yongwaizheng Street, Donghu District, Nanchang 330006, China. Email: 345936705@qq.com.

\begin{abstract}
Background: To evaluate functional connection density (FCD) in patients with diabetic optic neuropathy (DON) using the resting functional connectivity (FC) method, and to determine the abnormal areas of brain activity.

Methods: Patients with DON ( $\mathrm{n}=22 ; 10$ male, 12 female) and healthy controls (HCs; $\mathrm{n}=22 ; 10$ male, 12 female) were included in the study. The basic characteristics of the groups were matched. Functional magnetic resonance imaging (fMRI) was conducted with participants at rest, and long- and short-range FCD (long FCD and IFCD, respectively) were measured. Receiver operating characteristic (ROC) curve analysis was also conducted to determine whether DON and HC participants could be distinguished using fMRI indicators.

Results: Compared with HCs, the long FCD values of the left lingual gyrus, right lingual gyrus, right fusiform gyrus, and medial and lateral cingulate gyri were decreased in patients with DON. Further, the IFCD values of the left superior temporal gyrus, left inferior temporal gyrus, right inferior temporal gyrus, left cerebellar area 8, and right cerebellar Crus2 area were higher in patients with DON than in the HCs.

Conclusions: DON is associated with abnormal spontaneous brain activity. Our findings contribute to elucidating the mechanisms underlying the neuropathology of DON, and provide direction for further clinical research.
\end{abstract}

Keywords: Functional connectivity density (FC density); diabetic optic neuropathy spontaneous brain activity; functional magnetic resonance imaging (fMRI); diabetes

Submitted Mar 18, 2020. Accepted for publication Jan 13, 2021.

doi: 10.21037/qims-20-453

View this article at: http://dx.doi.org/10.21037/qims-20-453

\section{Introduction}

The incidence of diabetes as a chronic disease is increasing year by year. Of the complications caused by diabetes, the clinical manifestation of diabetic optic neuritis (DON) is among the more diverse. In severe cases, intraocular bleeding, cataracts, and retinal detachment may occur among other symptoms. As a disease involving the peripheral nerve, central nervous system, and sensory nerve, considerable variability in the reported incidence in clinical diagnosis, which makes the diagnosis challenging. Given that diabetic complications have become a leading cause of blindness, they deserve special attention. Freund first reported two patients with diabetes mellitus complicated with optic papilla edema in 1965 (1). Subsequently, 

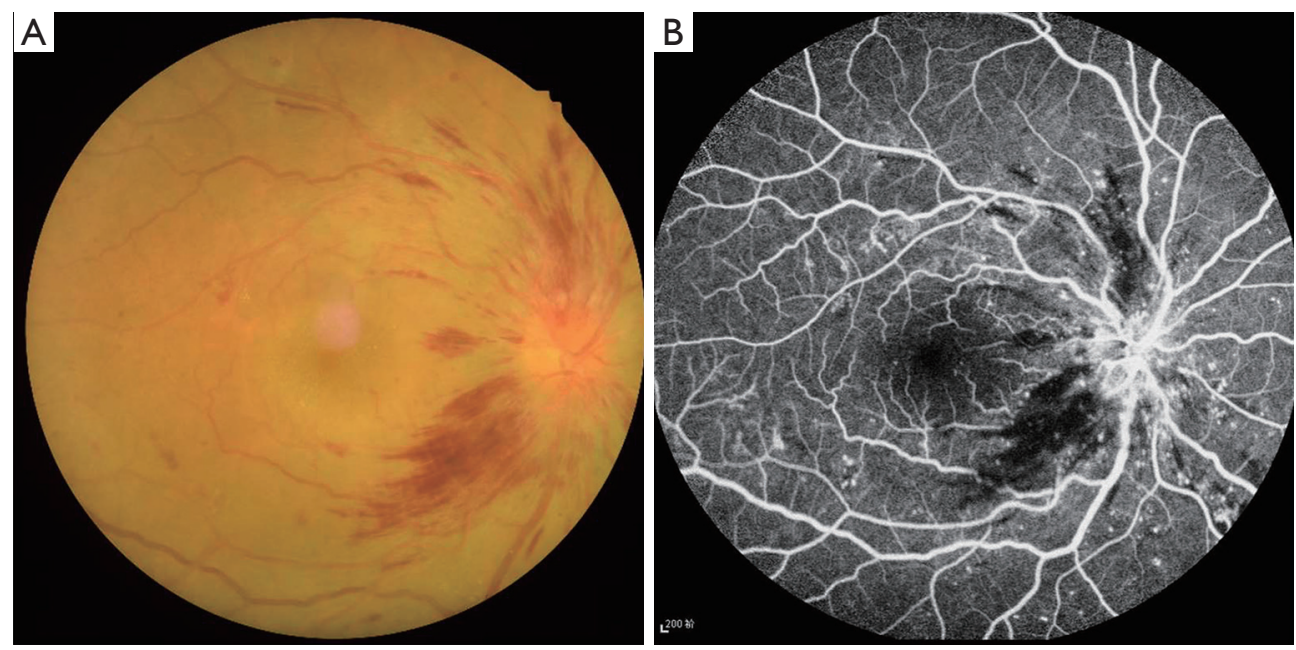

Figure 1 Examples of diabetic optic neuropathy (DON) in (A) fundus photograph and (B) on fundus fluorescein angiography.

in 1971, Lubow and Makley reported three cases of binocular papillary edema in adolescent patients with type 1 diabetes, which was then referred to as "diabetic pseudopapillary edema” (2). In 1980, Appen, Pavan, Barr, and others reported several groups of patients with type 1 diabetes, characterized by young age at onset and poor visual prognosis, and termed the condition "diabetic optic neuropathy" (3-5). Later, Bayraktar et al. discovered that patients with type 2 diabetes can also develop DON (6) (Figure 1).

The incidence of optic neuropathy in patients with diabetes varies widely. In a retrospective study of 3,235 patients with diabetes, Slagle et al. found that $45(1.4 \%)$ suffered from optic disc edema, of whom $16(0.49 \%)$ had diabetic optic papillopathy (diabetic papillopathy, $\mathrm{DP})$, and 19 (0.59\%) had ischemic optic neuropathy (7); however, some researchers have reported that the incidence of diabetes complicated with DON is as high as $48.3 \%$. It can be seen that DON shows a significant difference in reported morbidity. The variation in incidence with traditional clinical diagnostic methods may be due to (I) variations between studies in the research subjects; (II) lack of understanding of DON, leading to adoption of dissimilar diagnostic criteria and classification methods; (III) mild signs and symptoms of DON that are challenging to detect; or (IV) the ease with which signs and symptoms in cases complicated with diabetic retinopathy can be missed, resulting in missed or wrong diagnosis. As a new imaging examination method for use while the patient is in the resting state, brain functional magnetic resonance imaging (fMRI) can be used to detect changes in brain functional connectivity (FC) (8) and provides assistance and reference for clinical diagnosis. Compared with conventional MRI, fMRI offers more information about the activity of the cerebral cortex, allows precise localization, does not cause radiation damage, and facilitates the combination of functional and anatomical imaging. When the DON patient is examined, the functional state of the visual cortex and visual pathway can be noninvasively assessed. Therefore, resting fMRI has been successfully applied in the neurological studies of specific eye diseases (9-11).

Resting state FC (rsFC) analysis is an effective method of estimating spontaneous functional activity by FC and measuring the temporal correlation between oxygen leveldependent (BOLD) signals that occur sequentially in two brain regions. Long-distance FC density (longFCD) and shortdistance FCD (IFCD) can be calculated by mapping the FC density (FCD) of voxels in the whole brain, which helps to evaluate the functional information of the whole brain. Because alternate FCD can reflect spontaneous neural activity, it can be used to reveal the relationship between different brain regions. rsFC has been widely used to explore abnormal FCD changes in a variety of diseases, including Alzheimer disease (12), Parkinson disease (13), depression (14), and primary openangle glaucoma (15). We tried to use the resting FC method to evaluate functional connection density (FCD) in patients with diabetic optic neuropathy (DON), and to determine the abnormal areas of brain activity. 


\section{Methods}

\section{Subjects}

Patients ( $\mathrm{n}=22 ; 10$ male, 12 female) and healthy controls (HCs; $\mathrm{n}=22 ; 10$ male, 12 female), matched for sex, age, and education level, were randomly selected. All patients with DON were treated at the First Affiliated Hospital of Nanchang University. The diagnostic criteria for DON were as follows: (I) a clear history of diabetes mellitus; (II) optic disc edema (non-specific hyperemia and edema), optic disc neovascularization, or optic nerve atrophy and ischemic optic neuropathy; (III) different degrees of visual impairment, no clinical basis for visual impairment, no typical manifestations on visual field examination, and enlarged physiological blind spots or impairment limited to visual acuity; (IV) fundus fluorescein angiography revealing early lesions, involving part or all of the optic papilla or showing blurred, leaky, and low fluorescence; (V) exclusion of other diseases that may cause optic disc edema, such as hereditary optic neuropathy of Leber, toxic optic neuropathy and local ischemic papillary optic lesion, optic nerve trauma, congenital abnormalities of the optic nerve, or systemic lesions; and (VI) the disc edema caused by optic neuropathy which could be treated or would recover after approximately 6 months with the recovered disc may appearing pale; (VII) exclude other systemic disease, such as high blood pressure, etc. HCs were included if they met the following criteria: (I) no eye diseases; (II) no neurological or psychological illness; (III) MRI showing normal brain parenchyma; and (IV) clinically diagnosed with diabetes.

This study was approved by the Medical Ethics Committee of the First Affiliated Hospital. All methods used followed the tenets of the Declaration of Helsinki and complied with the principles of medical ethics. All subjects volunteered to participate in the study, understood its purpose, methods, and potential risks, and provided written consent for participation in the study.

\section{MRI data acquisition}

We used a 3.0 Tesla MR scanner (Siemens, Berlin, Germany) for all magnetic resonance imaging (MRI) examinations, and implemented $\mathrm{T} 1$ and $\mathrm{T} 2$ imaging sequences with the following parameters: echo time (TE) $=2.26 \mathrm{~ms}$, repetition time $(\mathrm{TR})=1900 \mathrm{~ms}$, gap $=0.5 \mathrm{~mm}$, thickness $=1.0 \mathrm{~mm}$, matrix $=256 \times 256$, field of view $(\mathrm{FOV}$ ) $=250 \times 250 \mathrm{~mm}$, flip angle $(\mathrm{FA})=9^{\circ}$, sagittal slices $=176$. In the resting state scanning session, we used a gradient echo- planar imaging sequence for 240 functional volumes under the following parameters: $\mathrm{TE}=30 \mathrm{~ms}$; $\mathrm{TR}=2,000 \mathrm{~ms}$; matrix $=64 \times 64$; slice gap $=1 \mathrm{~mm}$, and $\mathrm{FA}=90^{\circ}$. We also obtained 35 oblique slices, parallel to the anterior commissureposterior commissure (AC-PC) line by staggered acquisition. A sagittal fast spoiled gradient echo brain volume (FSPGR-BRAVO) sequence was used to obtain Tlweighted images with the following parameters: inversion time $(\mathrm{Tl})=450 \mathrm{~ms} ; \mathrm{TR}=8.208 \mathrm{~ms} ; \mathrm{TE}=3.22 \mathrm{~ms}$; voxel size $=0.5 \times 0.5 \times 1 \mathrm{~mm} ; \mathrm{FOV}=240 \times 240 \mathrm{~mm}$, and $\mathrm{FA}=120^{\circ}$.

\section{Data analysis}

MRIcro software (http://www.MRIcro.com) was used to assess data quality. To ensure the best possible stability, we also deleted the first 10 images. Remaining data were preprocessed using the Data Processing and Analysis for Brain Imaging (DPABI 2.1, http://rfmri.org/DPABI) toolbox, based on MATLAB 2010a (Mathworks, Natick, MA, USA). Participants were requested to remain still until the scan was completed. As recent research has shown that higher-order models benefit from elimination of head motion effects, we used the Friston 24-head motion parameters model to delete the potential impact of head motion (6 previously described head motion parameters and 12 corresponding square terms) $(16,17)$. Linear regression was used to remove variables from variation or movement effects from sources such as breathing, LF drift etc. After head motion correction, functional images were spatially normalized to Montreal Neurological Institute space. Next, we used band-pass filtering $(0.01-0.1 \mathrm{~Hz})$ and linear attenuation for the time series of each voxel to reduce the influence of breathing, low-frequency drift, linear attenuation of the time series, and cardiac noise.

\section{Construction of longFCD and IFCD maps}

longFCD and IFCD maps were used to assess gray matter. Based on the Pearson correlation between the time course of a given voxel and other voxels, we considered the number of functional connections of a given voxel as the nodal degree in the binary graph. We computed the values of FCD and longFCD using the Brainwaver toolbox (http://cran.rproject. org/src/contrib/Archive/brainwaver), and also estimated several connectivity metrics for each network node. The local efficiency of nodes was also estimated as an indicator suitable for evaluation of the performance of small-world brain functional networks (18). First, a correlation threshold $(r)>0.25$ was used to define the whole brain FC between a given voxel 
Table 1 Conditions of participants included in the study

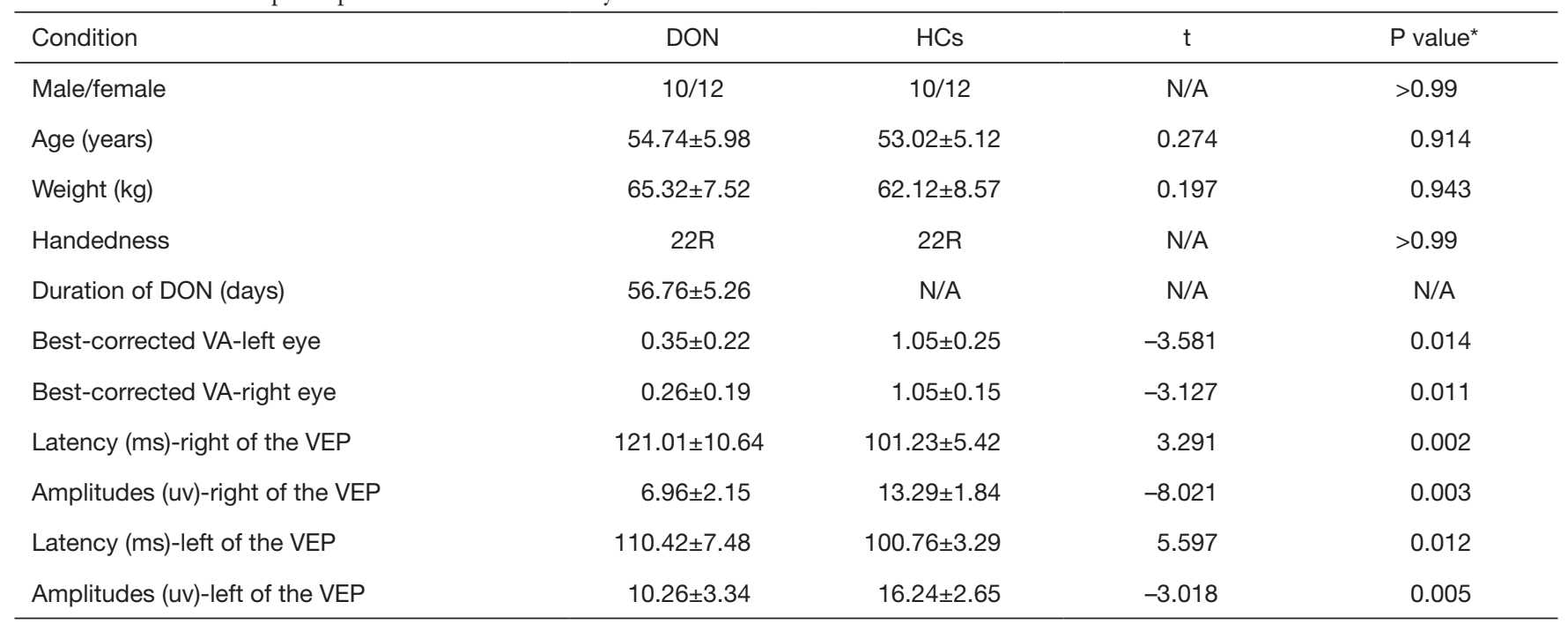

*, $\mathrm{P}<0.05$ independent $t$-tests comparing two groups. DON, diabetic optic neuropathy; HCs, healthy controls; N/A, not applicable; VA, visual acuity; VEP, visual evoked potential.

and other voxels. In the current study, those voxels that reached the threshold of $r>0.25$ within their neighborhood (anatomical distance $<14 \mathrm{~mm}$ ) were defined as IFCD voxels, while longFCD described those with an anatomic distance $>14 \mathrm{~mm}$. IFCD and longFCD maps were converted to $Z$-scores for normality enhancement. Finally, a $6 \times 6 \times 6 \mathrm{~mm}^{3}$ full-width Gaussian kernel was used to spatially smooth the IFCD and longFCD maps at half-maximum with statistical parameter mapping SPM8 (MathWorks, Inc., Natick, MA, USA).

\section{Ophthalmic testing}

All subjects were screened for visual acuity using the Snellen Vision Test at $5 \mathrm{~m}$, parallel to line 1.0 on the test.

\section{Statistical analysis}

An independent sample $t$-test (where a $\mathrm{P}$ value $<0.05$ represented a significant difference) and SPSS 16.0 software (IBM Corp. Armonk, NY, USA) were applied to analyze clinical variables. rsFC, longFCD, and IFCD values in each study group were compared, and the differences between patients and HCs were assessed using a onesample $t$-test $(\mathrm{P}<0.05)$. The SPM8 toolkit was used for linear model creation. Differences in cerebral blood flow plots between the patient and HC groups were assessed using a two-sample t-test [voxel level, Gaussian random field theory $(\mathrm{GRF})$ corrected $\mathrm{P}<0.01$ and $\mathrm{P}<0.05$ were considered statistically significant]. Mean rsFC, longFCD, and IFCD in different cerebral regions in the two groups were analyzed using receiver operating characteristic (ROC) curves. Pearson correlation $(\mathrm{P}<0.05$ considered statistically significant) was used to investigate the relationships among mean rsFC, long FCD, and IFCD in different cerebral regions of the patients and their clinical characteristics.

\section{Results}

\section{Demographic and visual measurements}

There were no significant differences in body weight, age, sex ratio, or handedness between the DON and HC groups; however, there were significant differences between left $(\mathrm{P}<0.014)$ and right $(\mathrm{P}<0.01)$ side best-corrected visual acuity (BCVA-L and BCVA-R, respectively). The average duration of DON was 56.76 \pm 5.26 days (Table 1).

\section{FCD analysis}

Compared with the values in the HCs, longFCD values of the left lingual gyrus (Lingual_L), right fusiform gyrus (Fusiform_R), right lingual gyrus (Lingual_R), and medial and lateral cingulate gyri (Cingulum_Mid_L) in the DON patients were significantly lower (Table 2). In addition, compared with the values in the HCs, IFCD values of the left cerebellar area 8 (Cerebelum_8_L), right 
Table 2 The binarized longFCD differences between DON patients and HCs

\begin{tabular}{|c|c|c|c|c|c|c|}
\hline Brain areas & \multicolumn{3}{|c|}{ MNI coordinates } & \multicolumn{3}{|c|}{ longFCD } \\
\hline \multicolumn{7}{|l|}{$\mathrm{HC}>\mathrm{DON}$} \\
\hline Lingual_L & -21 & -87 & -18 & 18 & 25 & 3.44 \\
\hline Fusiform_R & 36 & -72 & -18 & 19 & 59 & 4.11 \\
\hline Cingulum_Mid_L & -3 & -3 & 39 & 24 & 54 & 3.16 \\
\hline
\end{tabular}

Between-group differences in binarized longFCD at a threshold of $r=0.3$. We used thresholds of two-tailed voxel-wise $P<0.01$ and cluster-level $\mathrm{P}<0.05$, corrected for multiple comparisons by AlphaSim to determine the significant group differences. longFCD, long-range functional connectivity density; DON, diabetic retinopathy; HCs, healthy controls; R, right; L, left; BA, Brodmann's area; MNI, Montreal neurological institute.

Table 3 The binarized IFCD differences between DON patients and HCs

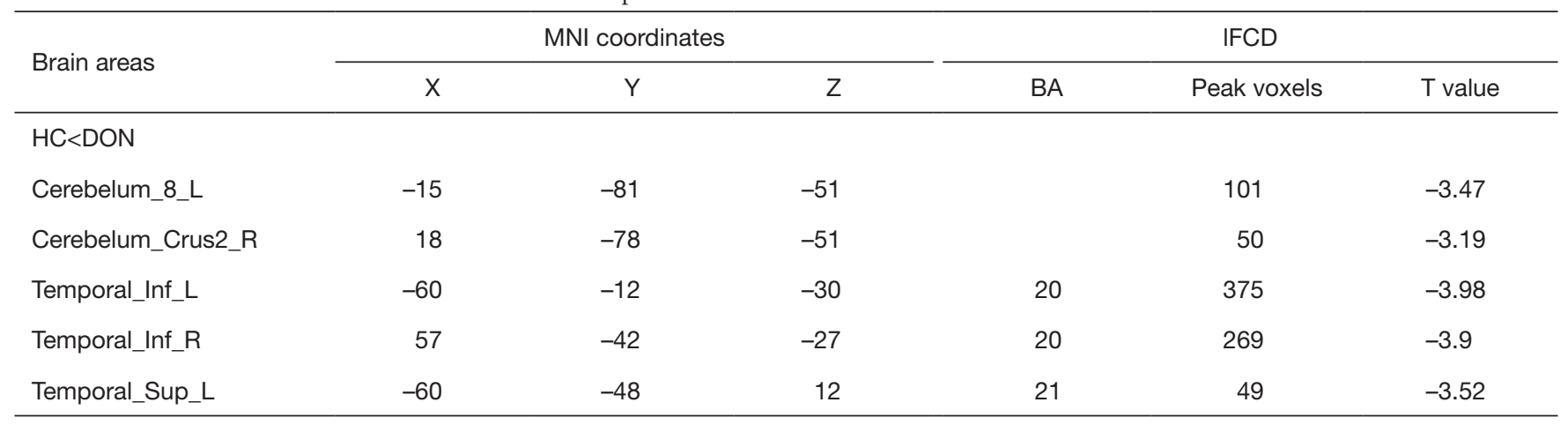

Between-group differences in binarized longFCD at a threshold of $r=0.3$. We used thresholds of two-tailed voxel-wise $P<0.01$ and cluster-level $\mathrm{P}<0.05$, corrected for multiple comparisons by AlphaSim to determine the significant group differences. IFCD, short-range functional connectivity density; DON, diabetic retinopathy; HCs, healthy controls; R, right; L, left; BA, Brodmann's area; MNI, Montreal neurological institute.

cerebellar Crus2 area (Cerebelum_Crus2_R), left inferior temporal gyrus (Temporal_Inf_L), right inferior temporal gyrus (Temporal_Inf_R), and left superior temporal gyrus (Temporal_Sup_L) in the patients with DON were significantly higher (Table 3, Figures 2-4).

\section{ROC curve analysis}

We hypothesized that the difference between longFCD and IFCD may be a feasible diagnostic biomarker to distinguish between the DON and HC groups. To test this, we used the ROC curve method to analyze the average FCD values of different brain regions. For the area under the curve (AUC), values between 0.5 and 0.7 indicated low precision, those between 0.7 and 0.9 indicated moderate precision, and values $>0.9$ indicated higher precision (18).
The AUC values for the Lingual_L, Fusiform_R, Lingual_R, and Cingulum_Mid_L were 0.787, 0.828, 0.764 , and 0.782 , respectively (Figure $5 A$ ) and those for the Cerebelum_8_L and Cerebelum_Crus2_R were 0.846 and 0.746 , respectively (Figure $5 A$ ). IFCD values for the Cerebelum_8_L and Cerebelum_Crus2_R were 0.846 and 0.746 , respectively. Finally, AUC values for the left infratemporal gyrus, right infratemporal gyrus, and left superior temporal gyrus were $0.773,0.841$, and 0.782 , respectively (Figure 5B).

We also analyzed the correlations between the value of each longFCD and IFCD and patient characteristics, including scores on the hospital anxiety, depression scale, and BCVA (Table 4). There were three significant correlations: the longFCD value of the left gyrus negatively correlated with BCVA-R $\left(\mathrm{R}^{2}=0.5387, \mathrm{P}<0.0001\right)$ (Figure 6A); 
A
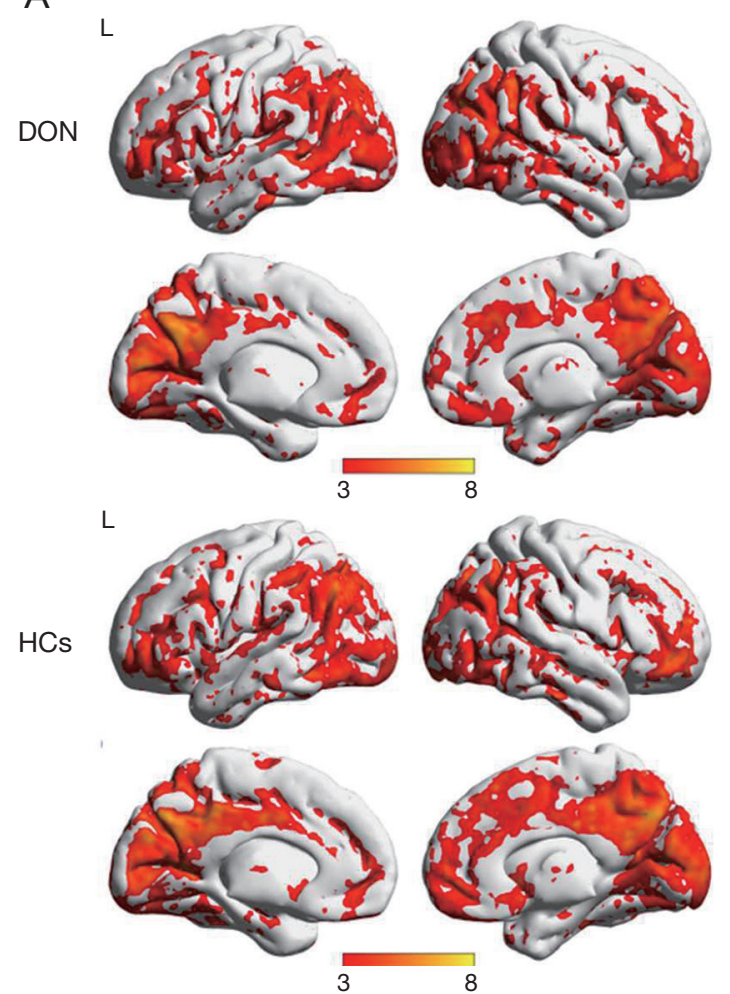

B
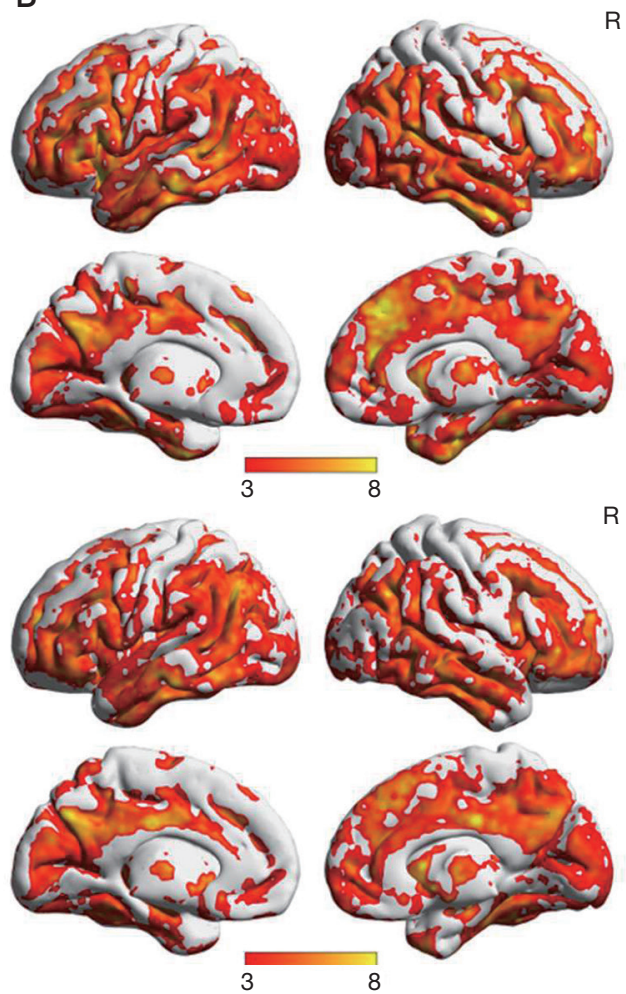

Figure 2 The red or yellow denotes higher longFCD and IFCD values. (A) Significant longFCD differences in the left lingual gyrus (Lingual_ L), right fusiform gyrus (Fusiform_R), right lingual gyrus (Lingual_R), and medial and lateral cingulate gyrus (Cingulum_Mid_L). The color bars show the means of altered longFCD between the DON and HC groups. (B) Significant IFCD differences in the left cerebellar area 8 (Cerebelum_8_L), right cerebellar Crus2 area (Cerebelum_Crus2_R), left inferior temporal gyrus (Temporal_Inf_L), right inferior temporal gyrus (Temporal_Inf_R), and left superior temporal gyrus (Temporal_Sup_L). The color bars show the means of altered IFCD between the DON group and HCs. DON, diabetic optic neuropathy; HC, healthy control; IFCD, short-range functional connectivity density; longFCD, long-range functional connectivity density; L, left; R, right.

the longFCD of the right gyrus negatively correlated with BCVA-L $\left(\mathrm{R}^{2}=0.6464, \mathrm{P}<0.0001\right)$ (Figure 6B); and the longFCD of the Cingulum_Mid_L negatively correlated with the score on the hospital anxiety and depression scale $\left(\mathrm{R}^{2}=0.385, \mathrm{P}<0.002\right)$ (Figure $6 C$ ).

\section{Discussion}

The pathogenesis of DON has yet to be fully elucidated. Most researchers believe that it is the result of a metabolic disorder caused by diabetes, toxicity of abnormal glucose metabolites, circulatory disturbance caused by the destruction of microvascular barriers, vascular leakage, reduced neurokinesis, hypoxia, and other factors. DP is the most important clinical symptom of DON. Its main pathogenic features include increased vascular permeability, leakage, and blockage of axial neurotransmitters by capillary lesions on and around the optic papilla. Ischemic optic neuropathy is mainly caused by insufficient supply of blood from the posterior ciliary artery, disordered vascular selfregulation, and decreased elasticity. In addition, increased blood viscosity and platelet aggregation increases the risk of ischemic optic neuropathy. Tissue hypoxia is the main factor leading to optic disc neovascularization (26). Zhao et al. compared the blood flow, vascular permeability, and pathological changes of optic nerve tissue between 10 diabetic and 10 healthy rats. Their results showed that blood flow in the optic nerve decreased, vascular permeability increased, and abnormal histopathological changes appeared in the diabetic rats; the histopathological 

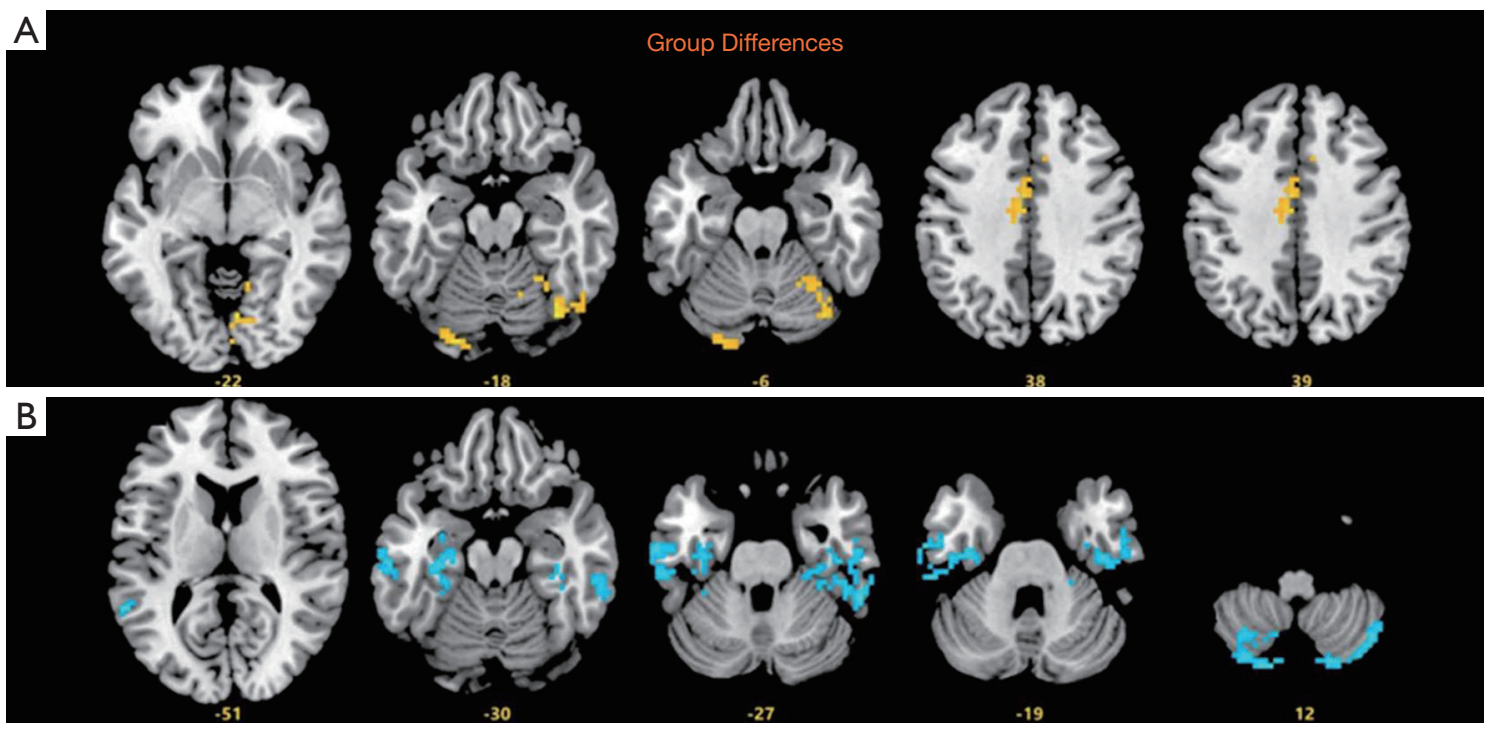

Figure 3 Binarized longFCD and IFCD differences between the HC and DON groups. The yellow and blue areas represent lower values. DON, diabetic optic neuropathy; HC, healthy control; IFCD, short-range functional connectivity density; longFCD, long-range functional connectivity density.
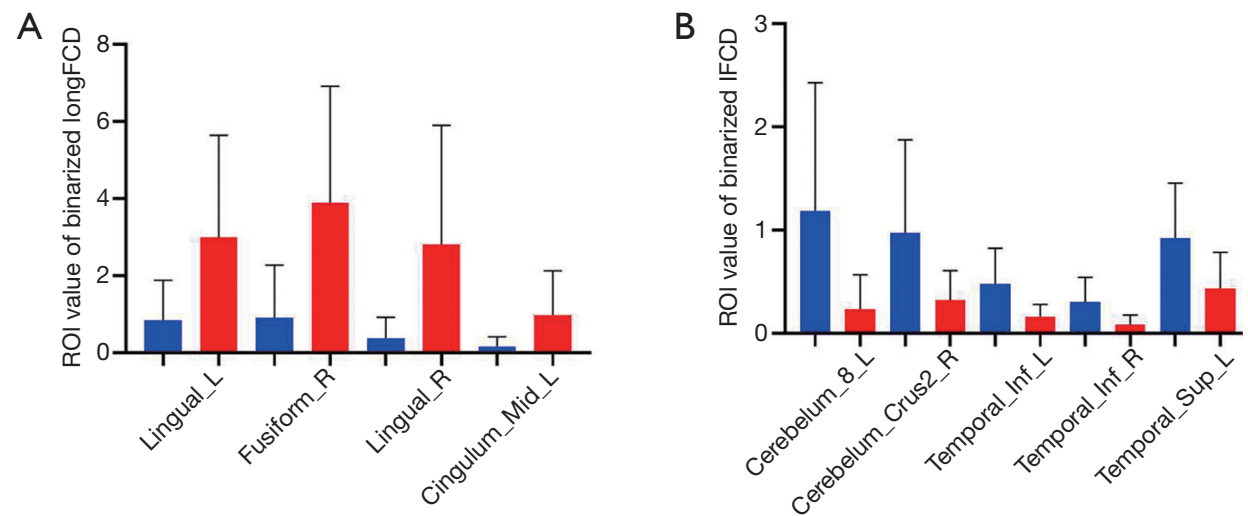

Figure 4 Binarized longFCD (A) and IFCD (B) ROI values in different brain regions. Red and blue bars indicate the HC and DON groups, respectively. DON, diabetic optic neuropathy; HC, healthy control; IFCD, short-range functional connectivity density; longFCD, longrange functional connectivity density; ROI, region of interest.

changes included myelin loss, package formation, abnormal astrocyte proliferation, nerve fiber atrophy, decreased vascular endothelial organelles, leukocyte aggregation, and adhesion to endothelial cells, among others (27).

At present, treatment of DON requires a comprehensive approach. First, it is necessary to control the primary disease and risk factors, such as diabetes, hypertension, and hyperlipidemia. Control of blood glucose is the main measure used to prevent DON. The longer the course of diabetes, the higher the risk of DON; therefore, the fundus of patients diagnosed with diabetes for $>10$ years should be examined regularly, with the aim of detecting DON and diabetic retinopathy at the earliest possible point. Some studies have reported that rapid control of blood glucose over a short period of time is a risk factor for DON (28); therefore, in patients with diabetes and high blood glucose levels, these should not be reduced too rapidly, rather blood glucose should be controlled in a gradual manner. People 

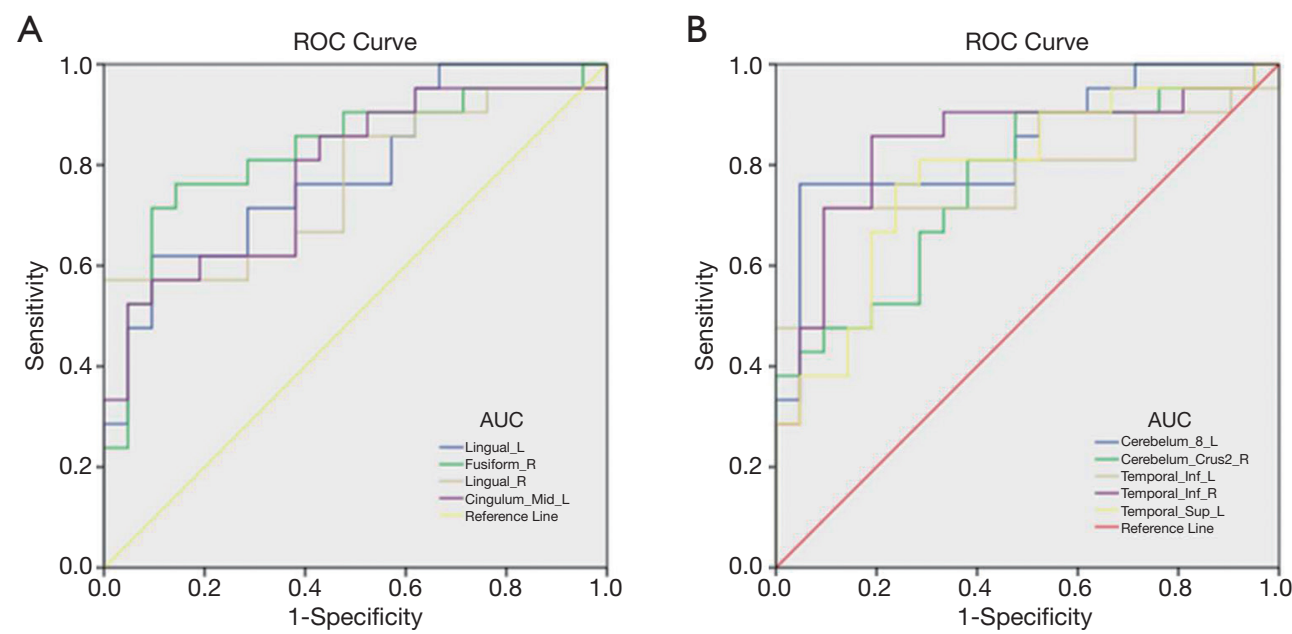

Figure 5 ROC curve analysis of the mean longFCD (A) and IFCD (B) values for different brain regions. The AUC values for the Lingual_ L, Fusiform_R, Lingual_R, and Cingulum_Mid_L were 0.787, 0.828, 0.764, and 0.782, respectively (A) and those for the Cerebelum_8_L and Cerebelum_Crus2_R were 0.846 and 0.746 , respectively (A). IFCD values for the Cerebelum_8_L and Cerebelum_Crus2_R were 0.846 and 0.746 . Finally, AUC values for the left infratemporal gyrus, right infratemporal gyrus, and left superior temporal gyrus were $0.773,0.841$, and 0.782 , respectively. AUC, area under the curve; IFCD, short-range functional connectivity density; longFCD, long-range functional connectivity density; ROC, receiver operating characteristic.

Table 4 FCD method applied in ophthalmological diseases

\begin{tabular}{lllll}
\hline Author & Year & Disease & Increase FCD & Decrease FCD \\
\hline Chen et al. (19) & 2019 & Primary angle-closure glaucoma & IFG PHG RMFG & CUN PoCG \\
Scheff et al. (20) & 2011 & Alzheimer's disease & - & ITG \\
Myung et al. (21) & 2016 & Adjustment disorder & - & MFG \\
Wang et al. (22) & 2014 & Anisometropic amblyopia & - & LIPL RpreCG LFFG LITG \\
Han et al. (23) & 2018 & Neuromyelitis optica & V1/V2 & BA11 LING \\
Zhai et al. (24) & 2016 & Pathological myopia & - & PCG RPCG LFFG RSMG \\
Tan et al. (25) & 2016 & Strabismus & - & MFG \\
Zhu et al. (10) & 2019 & corneal ulcer & MTG & MFG
\end{tabular}

IFG, inferior frontal gyrus; PHG, parahippocampal gyrus; RMFG, right middle frontal gyrus; CUN, cuneus; PoCG, postcentral gyrus; ITG, Inferior temporal gyrus; MFG, middle frontal gyrus; LIPL, left inferior parietal; RpreCG, right precentral gyrus; LFFG, left fusiform gyrus; LITG, left inferior temporal gyrus; LING, Lingual gyrus; PCG, posterior cingulate gyrus; RPCG, right posterior cingulate gyrus; RSMG, right supramarginal gyrus; MFG, middle frontal gyrus; MTG, middle temporal gyrus.

with a smaller optic disc cup to optic disc ratio are more likely to develop DP and ischemic optic neuropathy. Thus, particular attention should be paid to the examination of the optic nerve in patients with a small cup to disc ratio and diabetes.

We investigated functional brain alterations using rsFC. In the majority of previous studies, connections between brain regions were evaluated by selecting seed regions and considering their relationships with other brain areas. The FCD method does not need to assess relationships between brain regions or select seed points; rather, data are analyzed directly, leading to faster calculation speeds and results that are more accurate, as well as facilitating identification of important regions. The FCD method has been applied 
A

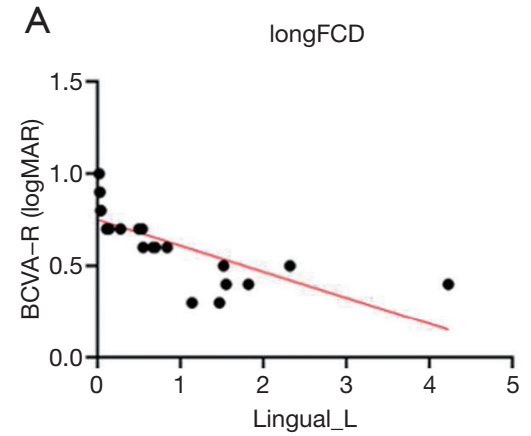

C

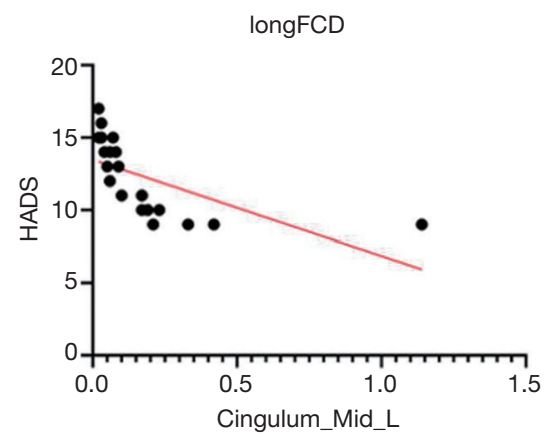

B

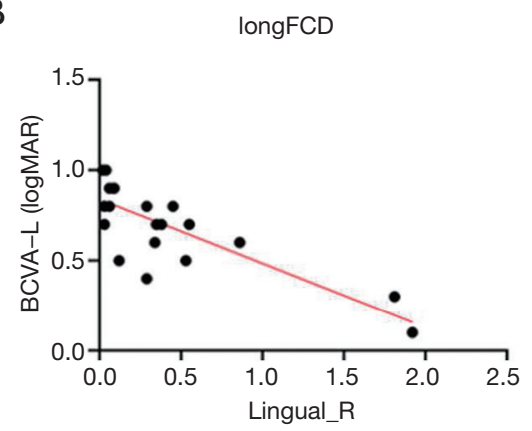

Figure 6 Correlations between the mean longFCD values and BCVA-R (logMAR). There was a negative correlation with the longFCD values of the Lingual_L, Lingual_R and Cingulum Mid_L. longFCD, long-range functional connectivity density; BCVA-R, best-corrected visual acuity of right eye.

in many research fields, including acquired blindness (29), anisometropic amblyopia (30), psychological phenanthrene seizures, Alzheimer disease (31), and adult chicken pox. Tomasi et al. found that local FCD is $14 \%$ higher in women than in men (32), demonstrating differences in FCD patterns between the sexes. Liu et al. (18) studied patients with schizophrenia and their siblings using an FCD approach. Their results showed that the density of FCs in the postcentral gyrus and suboccipital gyrus were significantly lower in patients with schizophrenia. Further, Zhu et al. (10) detected abnormal longFCD and IFCD values in several brain regions in patients with corneal ulcers.

There are no previous reports describing which brain areas exhibit changes in FCD associated with diabetic retinopathy. We therefore evaluated differences in longFCD and IFCD in 22 patients and $22 \mathrm{HCs}$, and explored the relationship between these indicators and patient symptoms. Compared with HCs, the longFCD values of the Lingual_L, Fusiform_R, Lingual_R, and Cingulum_Mid_L were significantly lower in patients with DON (Figure 7), while the IFCD values of the Cerebelum_8_L, Cerebelum_Crus2_R, Temporal_Inf_L, Temporal_Inf_R, and Temporal_Sup_L were significantly increased (Figure 7). Overall, we detected changes in spontaneous activity in multiple areas of the brain in patients with DON, contributing to our understanding of the pathogenesis of this condition.

The posterior part of the occipital lobe of the lingual gyrus is connected with the occipital pole, while the anterior side is connected with the surface of the temporal tentorium. The lingual gyrus has two major functions, one is visual processing-hence vision is affected by pathological changes to this area-and the other is language processing. Abnormal function of the gyrus of the tongue may affect responsiveness to external cues and cause problems with understanding. The cingulate gyrus is part of the limbic system cortex. In 1973, the emotion theory was proposed, which describes the anatomical basis of emotion as the papillary body, the anterior nucleus of the thalamus, the cingulate gyrus, the hippocampus, and their loops. The theory states that neural activity from 

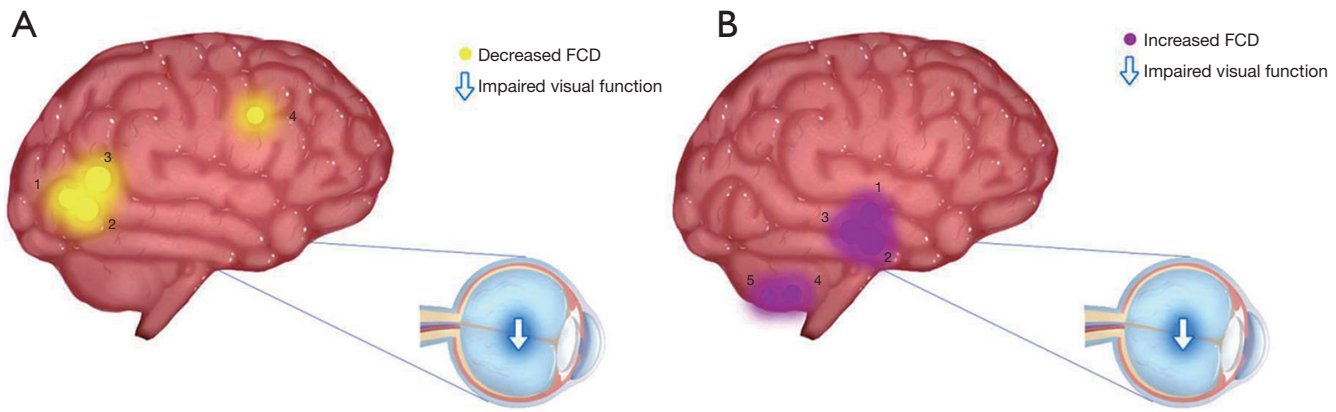

Figure 7 Mean longFCD and IFCD values of different brain regions in the DON group. Compared with HCs, longFCD values of the Lingual_L, Fusiform_R, Lingual_R, and Cingulum_Mid_L were significantly lower in patients with DON, while IFCD values of the Cerebelum_8_L, Cerebelum_Crus2_R, Temporal_Inf_L, Temporal_Inf_R, and Temporal_Sup_L were significantly increased. The numbers 1-5 indicate a node in a brain network. longFCD, long-range functional connectivity density; IFCD, short-range functional connectivity density; HCs, healthy controls; BA, Brodmann's area.

Table 5 Brain regions alternation and its potential impact

\begin{tabular}{llll}
\hline Brain regions & Experimental result & Brain function & Anticipated results \\
\hline Left/right lingual gyrus & DON $<\mathrm{HCs}$ & Visual processing & $\begin{array}{l}\text { Visual hallucination, hemianopsia in the } \\
\text { same direction, cortical blindness caused } \\
\text { by damage to the visual cortex of both } \\
\text { occipital lobes }\end{array}$ \\
Right fusiform gyrus & DON $<\mathrm{HCs}$ & Face recognition & $\begin{array}{l}\text { Mental visual impairment characterized } \\
\text { by visual distortion or agnosia }\end{array}$ \\
Medial and lateral cingulate gyrus & $\mathrm{DON}<\mathrm{HCs}$ & Body movement, body sensation & $\begin{array}{l}\text { Aphasia-aphasia-aphasia spirit } \\
\text { indifference }\end{array}$ \\
Cerebellar region & $\mathrm{DON}>\mathrm{HCs}$ & $\begin{array}{l}\text { Regulate muscle tone, maintain } \\
\text { coordination, maintain balance }\end{array}$ & $\begin{array}{l}\text { Ataxia, nystagmus } \\
\text { Left/right infratemporal gyrus }\end{array}$ \\
Left superior temporal gyrus & $\mathrm{DON}>\mathrm{HCs}$ & $\begin{array}{l}\text { Processing auditory information, } \\
\text { related to memory }\end{array}$ & Contralateral trunk ataxia \\
\hline
\end{tabular}

the neocortex representing an emotional process proceeds via the hippocampus, fornix, papillary body, and anterior nucleus of the thalamus, before finally projecting to the socalled emotional cortex. Primary cingulate gyrus epilepsy mainly manifests as complex partial seizures, accompanied by characteristic automatism, autonomic nerve dysfunction, emotional changes, and urinary incontinence. Resection of the cingulate gyrus to the epileptic focus and partial incision of the corpus callosum can effectively control epilepsy. Further, Zhu et al. (10) demonstrated that abnormal function of the cingulate gyrus is an imaging manifestation of depression. The fusiform gyrus is responsible for the secondary classification and identification of things. Opel et al. (33) showed that the gray matter volumes of the frontal lobe and orbitofrontal cortex in patients with depression were smaller than those in HCs, suggesting that frontal lobe and orbitofrontal cortex may be related to the pathogenesis of depression. Further, Cheng et al. (34) found that FC between the lateral orbitofrontal cortex and the $\mathrm{PC}$ is increased in patients with depression and associated with depressive symptoms, such as inferiority, despair, and lack of self-worth. Moreover, Shen et al. (35) showed that the cerebellar Crus2 region is associated with late-onset depression and early-onset depression in adulthood. The lower long-FCD values of the Cingulum and Cerebelum may therefore indicate abnormal activity in these brain regions and it can be associated with DON patients' pain and depression reaction (Table 5). 


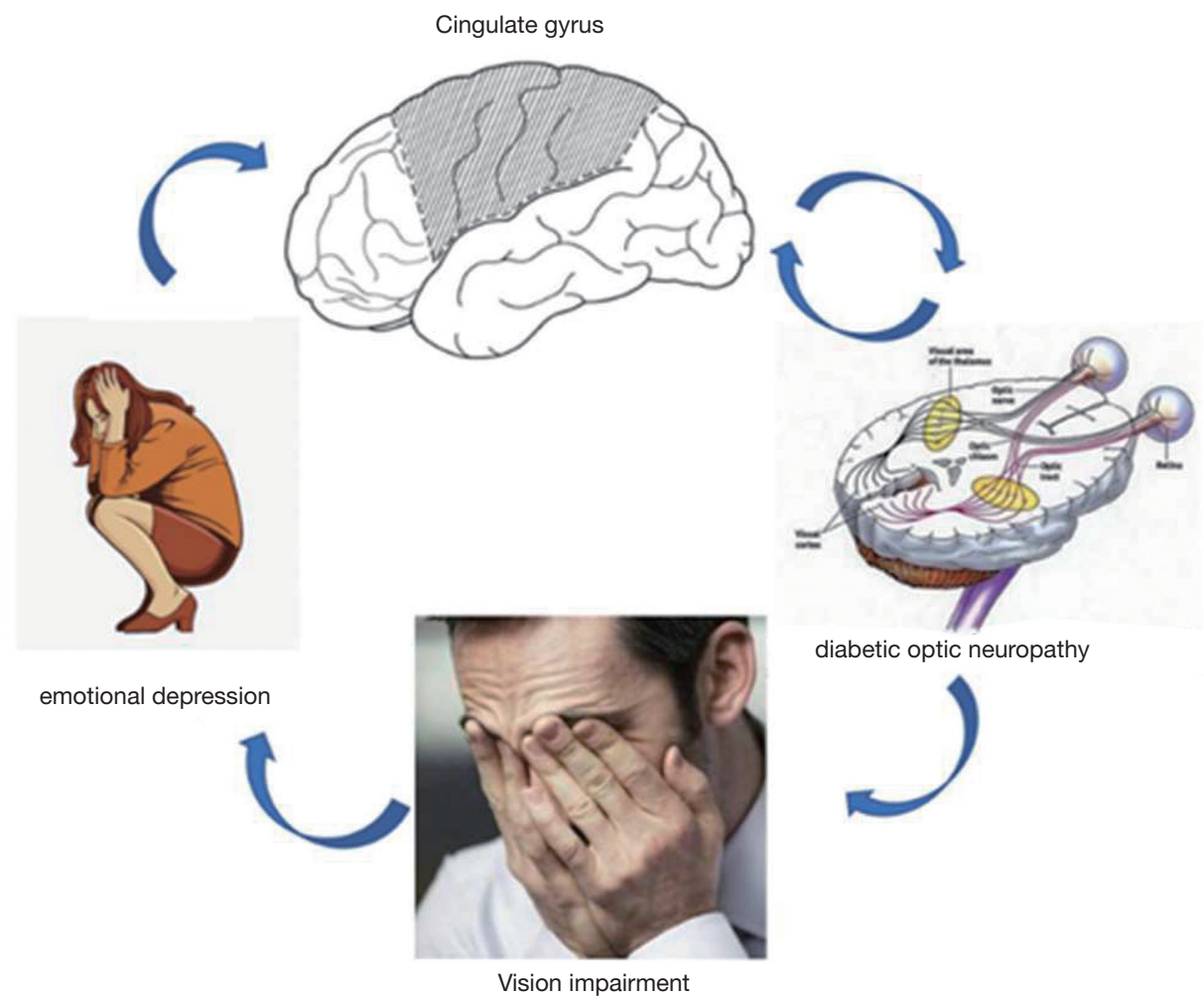

Figure 8 Schematic diagram of the relationship between diabetic retinopathy, FCD-related brain changes, and emotional depression. FCD, functional connection density.

DON can have serious detrimental consequences for patients, so it is imperative that it is diagnosed and treated at the earliest possible opportunity. However, the clinical manifestations of DON are variable, making diagnosis very challenging. Previous studies have found that the incidence of optic neuropathy increases with the progression of diabetes. Through analysis of brain FCD maps, we found that decreased longFCD values of the Lingual_L, Lingual_ R, Fusiform_R, and Cingulum_Mid_L, and increased IFCD values of the Temporal_Sup_L, Temporal_Inf_L, Temporal_Inf_R, Cerebelum_8_L, and Cerebelum_Crus2_ $\mathrm{R}$ are associated with diabetic retinopathy. This finding will be helpful for the assisted imaging diagnosis of DON, which will facilitate early diagnosis and treatment. At the same time, this also helps us to clarify the neuropathological mechanism of DON (Figure 8).

\section{Acknowledgments}

Funding: This study was supported by funding from the National Natural Science Foundation of China (No.
81660158). Natural Science Key Project of Jiangxi Province (No. 20161ACB21017), the Youth Science Foundation of Jiangxi Province (No. 20161BAB215198), the Key Research Foundation of Jiangxi Province (No. 20181BBG70004), the Excellent Talents Development Project of Jiangxi Province (No. S2019RCQNB0259), Teaching Reform of Degree and Graduate Education Research Project of Jiangxi Province (No. JXYJG-2018013).

\section{Footnote}

Conflicts of Interest: All authors have completed the ICMJE uniform disclosure form (available at http://dx.doi. org/10.21037/qims-20-453). The authors have no conflicts of interest to declare.

Ethical Statement: This study was approved by the Medical Ethics Committee of the First Affiliated Hospital. All methods used followed the tenets of the Declaration of Helsinki and complied with the principles of medical ethics. All subjects volunteered to participate in the study, 
understood its purpose, methods, and potential risks, and provided written consent for participation in the study.

Open Access Statement: This is an Open Access article distributed in accordance with the Creative Commons Attribution-NonCommercial-NoDerivs 4.0 International License (CC BY-NC-ND 4.0), which permits the noncommercial replication and distribution of the article with the strict proviso that no changes or edits are made and the original work is properly cited (including links to both the formal publication through the relevant DOI and the license). See: https://creativecommons.org/licenses/by-nc-nd/4.0/.

\section{References}

1. Freund M, Carmon A, Cohen AM. Papilledema and papillitis in diabetes: report of two cases. Am J Ophthalmol 1965;60:18-20.

2. Lubow M, Makley TA Jr. Pseudopapilledema of juvenile diabetes mellitus. Arch Ophthalmol 1971;85:417-22.

3. Pavan PR, Aiello LM, Wafai MZ, Briones JC, Sebestyen JG, Bradbury MJ. Optic disc edema in juvenile-onset diabetes. Arch Ophthalmol 1980;98:2193-5.

4. Appen RE, Chandra SR, Klein R, Myers FL. Diabetic papillopathy. Am J Ophthalmol 1980;90:203-9.

5. Barr CC, Glaser JS, Blankenship G. Acute disc swelling in juvenile diabetes. Clinical profile and natural history of 12 cases. Arch Ophthalmol 1980;98:2185-92.

6. Bayraktar Z, Alacali N, Bayraktar S Diabetic papillopathy intype II diabetic patients. Retina 2002;22:752-8.

7. Slagle WS, Musick AN, Eckermann DR. Diabetic papillopathy and its relation to optic nerve ischemia. Optom Vis Sci 2009,86:e395-403.

8. Shi WQ, Liu JX, Yuan Q, Ye L, Su T, Jiang N, Lin Q, Min YL, Li B, Zhu PW, Xu XW, Shao Y. Alternations of interhemispheric functional connectivity in corneal ulcer patients using voxel-mirrored homotopic connectivity: a resting state fMRI study. Acta Radiol 2019;60:1159-66.

9. Dong ZZ, Zhu FY, Shi WQ, Shu YQ, Chen LL, Yuan Q, Lin Q, Zhu PW, Liu KC, Min YL, Ye L, Shao Y. Abnormalities of interhemispheric functional connectivity in individuals with acute eye pain: a resting-state fMRI study. Int J Ophthalmol 2019;12:634-9.

10. Zhu F, Tang L, Zhu P, Lin Q, Yuan Q, Shi W, Li B, Ye L, Min Y, Su T, Shao Y. Resting-state functional magnetic resonance imaging (fMRI) and functional connectivity density mapping in patients with corneal ulcer. Neuropsychiatr Dis Treat 2019;15:1833-44.
11. Zhu PW, Chen Y, Gong YX, Jiang N, Liu WF, Su T, Ye L, Min YL, Yuan Q, He LC, Shao Y. Altered brain network centrality in patients with trigeminal neuralgia: a restingstate fMRI study. Acta Radiol 2020;61:67-75.

12. Hata M, Kazui H, Tanaka T, Ishii R, Canuet L, PascualMarqui RD, Aoki Y, Ikeda S, Kanemoto H, Yoshiyama $\mathrm{K}$, Iwase M, Takeda M. Functional connectivity assessed by resting state EEG correlates with cognitive decline of Alzheimer's disease - an eLORETA study. Clin Neurophysiol 2016;127:1269-78.

13. Hu X, Jiang Y, Jiang X, Zhang J, Liang M, Li J, Zhang Y, Yao D, Luo C, Wang J. Altered functional connectivity density in subtypes of Parkinson's Disease. Front Hum Neurosci 2017. doi: 10.3389/fnhum.2017.00458.

14. Maglanoc LA, Landrø NI, Jonassen R, Kaufmann T, Córdova-Palomera A, Hilland E, Westlye LT. Data-driven clustering reveals a link between symptoms and functional brain connectivity in depression. Biol Psychiatry Cogn Neurosci Neuroimaging 2019;4:16-26.

15. Dai H, Morelli JN, Ai F, Yin D, Hu C, Xu D, Li Y. Resting-state functional MRI: functional connectivity analysis of the visual cortex in primary open-angle glaucoma patients. Hum Brain Mapp 2013;34:2455-63.

16. Satterthwaite TD, Elliott MA, Gerraty RT, Ruparel K, Loughead J, Calkins ME, Eickhoff SB, Hakonarson H, Gur RC, Gur RE, Wolf DH. An improved framework for confound regression and filtering for control of motion artifact in the preprocessing of resting-state functional connectivity data. Neuroimage 2013;64:240-56.

17. Yan CG, Cheung B, Kelly C, Colcombe S, Craddock RC, Di Martino A, Li Q, Zuo XN, Castellanos FX, Milham MP. A comprehensive assessment of regional variation in the impact of head micromovements on functional connectomics. Neuroimage 2013;76:183-201.

18. Liu C, Zhang W, Chen G, Tian H, Li J, Qu H, Cheng L, Zhu J, Zhuo C. Aberrant patterns of local and longrange functional connectivity densities in schizophrenia. Oncotarget 2017;8:48196-203.

19. Chen LL, Li SH, Cai FQ, Wu L, Zhou FQ, Pei ZG, Zeng $\mathrm{XJ}$. Changes in brain functional connectivity density in primary angle-closure glaucoma based on resting state fMRI. Journal of Clinical Radiology 2019;38:2243-7.

20. Scheff SW, Price DA, Schmitt FA, Scheff MA, Mufson EJ. Synapticloss in the inferior temporal gyrus in mild cognitive impairment and Alzheimer's disease. J Alzheimers Dis 2011;24:547-57.

21. Myung W, Na KS, Ham BJ, Oh SJ, Ahn HW, Jung HY. Decreased medial frontal gyrus in patients with adjustment 
disorder. J Affect Disorders 2016;191:36-40.

22. Wang T. Study on brain functional connection density of children with anisometropia amblyopia based on resting state fMRI. Tianjin Medical University; 2014.

23. Han YL, Li Y, Luo Q, Fu J, Kong L, Xiang Y, Chen X, Gu $\mathrm{Y}$. Study on resting state $\mathrm{fMRI}$ of functional connection density changes in patients with optic neuromyelitis. Magnetic Resonance Imaging 2018;9:33-7.

24. Zhai L. Changes of brain functional connectivity in high myopia based on resting state fMRI. Tianjin Medical University; 2016.

25. Tan G, Huang X, Ye L, Wu AH, He LX, Zhong YL, Jiang N, Zhou FQ, Shao Y. Altered spontaneous brain activity patterns in patients with unilateral acute open globe injury using amplitude of low-frequency fluctuation: a functional magnetic resonance imaging study. Neuropsychiatr Dis Treat 2016;12:2015-20.

26. Almog Y, Goldstein M. Visual outcome in eyes with asymptomatic optic disc edema. J Neuroophthalmol 2003;23:204-7.

27. Zhao JP, Ma ZZ, Song C, Li XH, Li YZ, Liu YY. Optic nerve lesions in diabetic rats: blood flow to the optic nerve, permeability of micro blood vessels and histopathology. Int J Ophthalmol 2010;3:291-4.

28. Zhang W, Nie H. Diabetic optic neuropathy. Chinese Journal of Practical Nervous Diseases 2016;19:135-7.

29. Qin W, Xuan Y, Liu Y, Jiang T, Yu C. Functional Connectivity Density in Congenitally and Late Blind Subjects. Cereb Cortex 2015;25:2507-16.

Cite this article as: Chen SY, Cai GQ, Liang RB, Yang QC, Min YL, Ge QM, Li B, Shi WQ, Li QY, Zeng XJ, Shao Y. Regional brain changes in patients with diabetic optic neuropathy: a resting-state functional magnetic resonance imaging study. Quant Imaging Med Surg 2021;11(5):21252137. doi: $10.21037 /$ qims-20-453
30. Wang T, Li Q, Guo M, Peng Y, Li Q, Qin W, Yu C. Abnormal functional connectivity density in children with anisometropic amblyopia at resting-state. Brain Res 2014;1563:41-51.

31. Tomasi D, Volkow ND. Aging and functional brain networks. Mol Psychiatry 2012;17:471-549.

32. Tomasi D, Volkow ND. Gender differences in brain functional connectivity density. Hum Brain Mapp 2012;33:849-60.

33. Opel N, Redlich R, Dohm K, Zaremba D, Goltermann J, Repple J, Kaehler C, Grotegerd D, Leehr EJ, Böhnlein J, Förster K, Meinert S, Enneking V, Sindermann L, Dzvonyar F, Emden D, Leenings R, Winter N, Hahn T, Kugel H, Heindel W, Buhlmann U, Baune BT, Arolt V, Dannlowski U. Mediation of the influence of childhood maltreatment on depression relapse by cortical structure: a 2-year longitudinal observational study. Lancet Psychiatry 2019;6:318-26.

34. Cheng W, Rolls ET, Qiu J, Liu W, Tang Y, Huang CC, Wang X, Zhang J, Lin W, Zheng L, Pu J, Tsai SJ, Yang AC, Lin CP, Wang F, Xie P, Feng J. Medial reward and lateral non-reward orbitofrontal cortex circuits change in opposite directions in depression. Brain 2016;139:3296-309.

35. Shen $Y$, Wei Y, Yang XN, Zhang G, Du X, Jia Q, Zhu X, Ma Y, Lang X, Luo X, Zhang XY. Psychotic symptoms in first-episode and drug naïve patients with major depressive disorder: Prevalence and related clinical factors. Depress Anxiety 2020;37:793-800. 